\title{
THE NEW YORK ACADEMY OF MEDICINE.
}

\author{
SLC'ION ON OTOLOGY.
}

Regular Mecting Octobev 13, 192?.

\section{Radical Mastoidectomy; Complete Healing; Method of Controlling Per- sistent Vertigo. Dr. Jacob L. Maybaum.}

Hugh B., age 26 year's, was admitted to Manlattan Dye, Ear and Throat Hospital, service of Dr. Berens, Jume 10, 1919, with a history of distressing vertigo and falling of six montlis' duration.

The patient had the usual diseases of childhood. Double middle ear suppuration dated back thirteen years following diphtheria. From then until the onset of vertigo six months ago, except for aural discharge and impaired hearing, there was no complaint of the ears. The dizzy attacks at the outset, occured at infrequent intervals, until six months before entering the hospital. The attacks then became of greater severity, occurring daily and being accompanied by falling, particularly to the right.

Aural examination disclosed a moderate, foul smelling discharge from each middle ear with perforation and granulations in posterior superior quadrant. Fearing in the right ear malkedly diminished, whisper not heard, $\mathrm{C}$ (128) and $\mathrm{C}+$ tuning forks not heard, $\mathrm{C}-\mathrm{A}=4$ sec. $\mathrm{B}$. 15 sec. Weber to the right. Mixed form of deafness-alvanced middle ear deafness with degenerative cochlear changes.

Hearing in the left ear fairly good-whisper 8 feet; Rinne negative; high tones as well as the low and middle tones slightly reduced.

There was a spontaneous nystagmus to the right, occasionally to the left, no spontaneous past-pointing. Fistula test negative.

Turning to right-horizontal nystagmus to the left 20 sec. Turning to left-nystagmus to right 22 sec.

Caloric stimulation of the right and left horizontal and vertical canals gave normal responses for nystagmus, past-pointing and vertigo.

I did a radical mastoid operation on the right side. Sclerosed mas. toid. Cholesteatomatous mass in antrum and aditus and granulations in middle ear removed. The patient left the hospital on the tenth day decidedly better, but still complained of occasional milder attacks of vertigo.

The spontaneous nystagmus disappeared at the end of two weeks, the hearing in the right ear remained as before the operation. The patient came to the clinic for two or three dressings; did not appear again for six weeks. He then returned with the story of having been unable to attend the clinic because of severe attack of acute articular rheumatism. During this period the ear had been dressed in a more or less perfunctory manner by his family physician. The vertiginous symptoms had returned with almost as marked an intensity as jrevious to the operation.

Examination of the operated ear now disclosed an almost complete atresia of the meatus, the canal was flled with partly organized granulation and connective tissue. There was a fistula in the postero-inferior region of the canal through which a thin, purulent, foul-smelling discharge was escaping.

Fearing very much impaired; could lear only loudly spoken voice with noise apparatus in left ear. Tuning forks not heard, Galton whistle heard.

Caloric test (right ear) ineffective because of the partial atresia of the canal. Caloric (left eal') normal response. A combined spontaneous nystagmus to the right present. Turning to the right ten times in twenty seconds resulted in horimontal nystagmus to left of 18 seconds' duration. Turning to the left nystagmus to the right 20 seconds' duration.

Patient was readmitted to the hospital. I reopened the post-auricular wound. Curetted granulation in meato-mastoid cavity and dirl a plastic operation. 
Patient left the hospital in a fow days; meato-mastoid cavity completely epidermatized in eight weeks. Until the end of May, 1922, the patient continued to complain of vertigo; at times this became so distressing that he had to discontinue his usual activities. Enforced rest gave only temporary relief. The symptoms described by the patient were definitely those of a systematized vertigo. Occasionally these attacks were accompanied by nausea, but never vomiting. The spontaneous nystagmus ceased two weeks after the second operation and was not again observed.

Functional examination October 11, 1922. Right o

Cr-A: 8 sec. B: 25 sec. Other foriks not heard. Voice heard with noise apparatus in left ear. Caloric positive in 35 sec.

\author{
Left \\ 15 feet \\ Whisper Lateralizes \\ C-A 115 B: 25 \\ $\mathrm{C}_{4}-6$ sec. \\ Caloric not performed \\ (dry perforation)
}

Turning to $R$ horizontal nystagmus to L. 22 sec.

Turning to $L$. horizontal nystagmus to $R$. 18 sec.

Every possible factor causing these attacks of vertigo were considered in order to give relief to the patient.

1. A continuance of irritation from a para or perilabyrinthine condition in the operated ear was constantly kept in mind.

2. A paralabyrinthine inflammation in the non-operated (left ear) was for a time seriously considered. Because of the marked diminution of hearing in the operated (right ear), I was loathe to seriously regard this factor from the standpoint of operative interference. The presence of comparatively good hearing in this ear (whisper 8 feet), together with absence of spontaneous nystagmus pointed against the left ear as a causative factor. Fistula test negative. Under treatment this ear has further improved (hearing now whisper $15 \mathrm{ft}$.); there has been little, at times no discharge cluring the past year.

3. Both vestibular nerves and their intracranial pathways gave normal responses for nystagmus, past-pointing and vertigo.

4. Cerebro-spinal lues has been ruled out. Wassermann being negative.

5. Other extraneous factor's; as local infection, gastro-intestinal disease, errors of refraction were carefully considered and eliminated as causes.

6. Vaso-motor disturbance of the labyrinth. After unsuccessfully running through the gamut of the various remedies advised for the relief of these patients, such as small doses of quinin, large doses of bromides, pilocarpin, etc., in an empirical way, I began to use of solution adrenalin $1 / 1000$ - ten dorps b. i. d. by mouth as suggested by Vernet in his article "The treatment of vertigo by means of Adrenalin," appearing in the March, 1922, issue of TrE LAmY NGoscorts. In a few days the patient obtained complete relief from his annoying symptoms. Withdrawal of the adrenalin for a few days after its administration for ten days was followed by a return of vertigo, but to a lesser degree. Adrenalin administration was interupted every ten days. During the past two weeks adrenalin medication has been discontinued with no recurrence of vertigo. His general condition has steadily improved during the past few months.

Chronic Middle Ear Suppuration Complicated by an Acute Suppurative Labprinthitis; Meningitis Sympathica; Labyyrinthectomy; Recovery. Dr. I. Friesner and Dr. J. L. Maybaum, New York, N. Y.

A careful decision as to the time of operation is of utmost importance in determining the outcome of a case of suppurative labyrinthitis.

On the one hand there are those favorably disposed to prompt surgical Interference during the acute stage of the disease or with the onset of symptoms of meningeal involvement. The majority of otologists in this country regard the layent stage the ideal time for labyrinthine surgery.

A labyrinthectomy too early performed, before barriers against spread of infection have been erected, usually results in a diffuse purulent men- 
ingitis. Meningeal involvement occurring during the course of an acute suppurative labyrintlitis is not necessarily an indication for immediate surgical interference. The clinical course and repeated spinal fluid examinations are especially helpful in arriving at a decision. $A$ persistent high count in the spinal fluid, together with clinical evidence, for op-
eration.

The problem of so important a decision confronted us in this case.

Anna H., age 26 years, was admitted to the ear service of Dr. Friesner of Mt. Sinai Hospital January 29, 1922, complaining of intense general. ized headache, rigidity of the neck of four days' duration.

Three years ago the patient had a severe attack of influenza, during the course. of which she developed an acute purulent otitis media on the left side. From then until last Christmas, except for moderate purulent discharge and slightly impaired hearing, the patient had had no symptoms referable to the ear. At this time, without any assignable cause, she began to vomit, complained of severe vertigo and staggered on attempting to walk. The tendency to stagger and fall was not in any definite direction. There was no history of pain, fever or chills. The attack continued for three days, the symtoms lessening in severity and entirely disappearing at the end of this period. Two weelts later, about January 7, the patient had a recurrence of her former symptoms and in addition intense pain in her left ear, which subsided the following day. This attack, as in the previous instance, lasted three days. During the next three weeks the patient was quite free from symptoms. On admission to the hospital she complained of intense lancinating pain in the head and rigidity of the neck of four days' duration. She had no pain in the ears, no nausea, vomiting, staggering or dizziness as on the previous occasions. Temperature on admission $105^{\circ}$, pulse 100 , respiration 28 .

Examination of the left ear disclosed a chronic middle ear suppuration. There was a large granuloma springing from a perforation in Shrapnell's membrane; a moderate foul smelling purulent discharge from the middle ear, no evidence of an acute exacerbation. No tenclerness or thickening over the mastoid. The right ear is normal.

Functional examinations disclosed a complete loss of labyrinthine function on the left side. There was a total deafness and complete loss of vestibular reaction on caloric stimulation. Fistula test negative.

The patient had marked rigidity of the neck. There was no Kernig or Babinski. Mentality clear. Ocular fundi normal. Four days later evidence of a left-sided facial paresis began to assert itself.

The temperature from $105^{\circ}$ on admission gradually came down to $100^{\circ}$ in five days and remained between $99^{\circ}$ and $100^{\circ}$ during the next three days. The patient vomited at times. Heaclache and rigidity of neck les. sened decidedly. Two days later there occurred a distinct rise of tem. perature from $99^{\circ}$ to $105^{\circ}$; this was repeated on two strcceeding days. The temperature then remained between $100^{\circ}$ and $101^{\circ}$. Pulse throughout about 1.00 .

Four lumbar punctures were made with these interesting findings:

January 29. Spinal fluid slightly cloudy, under moderately increased pressure. 4400 cells to the $\mathrm{cm}$. 90 per cent polys. Smear showed gram positive extra-cellular diplococci-two to the field. Could not be grown
in culture.

January 30. 6400 cells count, 90 per cent polys., no organisms on smear or culture.

January 31. Turbid fluid under moderately increased pressure. 1060 cells per cm. m., 90 per cent polys.

February 7. 1200 cells with an excess of lymphocytes. Fluid not quite so turbicl as previous specimens. Blood culture negative.

1. 29. 22: W. B. C. 12800 ; polys 94 per cent, lymphocytes 6 per cent.

2. 9. $22:$ W: B. C. 14000 ; polys 87 per cent, lymphocytes 13 per cent.

With the above history, aural examination, laboratory findings, a diagnosis of acute suppurative labyrinthitis with meningeal involvement was made. We hesitated to call this a bacterial meningitis based on one laboratory. finding-and this only a smear. The treatment, however, was 
clearly indicated-nothing less than a radical mastoldectomy and a labyrinthectomy. The question, however, as to the time of operation was one of utmost importance. We decided to temporize for the time being in order to permit the inflammatory process in and about the labyrinth to become localized. The first spinal fluid examination, indicating the pres. ence of a bacterial meningitis, pointed to a hopeless condition.

At the expiration of two weeks from the time the patient was admitted, we decided to operate. Patient was then having temperature. I did a radical mastoidectomy. Mastoid schlerotic throughout. Cholesteatoma in the antrum and aditus. A fistula could be seen in the horizontal canal. Dr. Friesner then did a labyrinthectomy. This consisted of thoroughly exposing and uncapping the three semi-circular canals, opening the vestibule and uncapping the cochlea. While uncovering the horizontal canal, the necrosed lower lip became loose, resulting in an exposure of the facial nerve above the oval winclow for a distance of about one-half inch. The paresis, no doubt, had been due to pressure or irritation from the necrotic area of bone.

The patient reacted very well following the operation. Headnche ceased entirely after forty-eight hour's and did not recur. To our great surprise and gratification the exposure of the nerve did not result in a facial paralysis; on the contrary, the paresis which had been previously present rapidly improved and is now no longer evident.

After the first full dressing, Dakin's solution was used throughout. A plastic operation was not done. Patient left the hospital March 24, 1922, relievel of the serions condition for which she had come.

The salient fentures of this case are:

1. Chronic midlle ear suppuration: two distinct attacks of labyrinthine disturbance; acute purulent labyrinthitis; meningitis sympathica.

2. Development of a facial paresis before operation; rapidly disappeared in spite of exposure of the nerve at the lower lip of the horizontal canal.

3. Laboratory findings in the spinal fluid characteristic of a meningitis sympathica.

4. Decision as to the time of operation.

5. Prompt disappearance of all symptoms following a labyrinthectomy.

Chronic Suppurative Otitis Media; Radical Mastoidectomy; Acute Suppurative Labyrinthitis; Serous Meningitis; Labyrinthectomy; Recov. ery. Dr. Mark J. Gottlieb.

Mrs. I. J. October 10, 1921. Aged 26 years. Married.

Has had a discharge from the right ear since childhood. The discharge has been intermittent, there being long periods when it was not evident. For the past eight months, the ear lias been discharging constantly, but not profusely. The patient has had headaches most every day, and dizziness occasionally.

'There is a large perforation of the inferio-posterior portion of the right drum, and the discharge, although scant, is fetid. Spreads of the discharge l'eveal Gram-positive cocci, epithelial cells, and no cholesteriue crystals. All the attachments of the muscles at the base of the skull, including the sterno-cleido-mastoid, were exquisitely tencler. The tonsils were large and the crypts contained many cheesy concretions. An examination of the hearing revealed that the Weber was referred to the right, the low tone limit of the right ear was raised above forty-eight double vibrations. The Rinne was negative. Conversational voice, nine feet, as comprared to the left, which was forty feet. Whispered voice was heard three feet with the right ear als compared with the leit, fifteen feet. Tho high tones were heard equally with both ear's.

In consideration of the fact that the right ear was a serviceable organ by virtue of the result of the hearing test, and the tonsils were diseased, probably being the cause of all the symptoms, the tonsils were removed under ether anesthesia and in a comparatively short time the beadacho and occasional dizziness disippeared.

About four months later, the patient complained of hendache, slight vertigo, nausea, and when the discharge from the right ear ceased, the 
patient felt drowsy. When the ear began to run again, the drowsiness disappeared.

Examination of the vestıbular apparatus cemonstrated that she pastpointed sponcuneousiy to a singht degree witn the leit arm to the right, and un attempt to overthrow vackward, the pelvic girdle reaction was not prompt. There were no ocher spontaneous pnenomena. un turning to the riglit with the horizontal semi-circular canals parallel to the floor, she developed a liuge nystugnus of twenty-five seconds' duration, to the left. The same result was obtained on rotation to the left, except that the nystagmus was to the right. On rotation to the right, with the vertical canals on the horizontal plane, she fell markedly to the right. On rotation to the left in the same position, she fell slightly to the left. On lotation to the right, she past-pointed twelve inches to the right with the right hand, and with the left past-pcinted slightly to the left, and then four inches to the right. On rotation to the left, the past-pointing was found to be normal. Caloric reaction was not performed.

The X-ray made by Dr. Frederick M. Law was as follows:

The right mastoid is undeveloped and shows some sclerosis around the knee. There is a small area below the linee, between the anterior wall of the sinus and the posterior canal wall, which shows bone absorption.

The region around the antrum shows organized tissue, with some bone absorption below the tegmen which suggests cholesteatoma.

This is evidently a chronic ear with some bone absorption.

The left mastoid is small and clear.

In view of these findings, it was felt that when the discharge from her right ear stopped it accumulated and caused a pressure symptoms, and a radical mastoid operation was performed. The bone was very hard and very few cells were encountered. The mastoid antrum and the area between the posterior wall and the lateral sinus contained cholesteatomatous material. The sinus plate was necrotic at the knee, and the dura of the middle fossa was exposed for an area about the size of a dime. The dura was opaque and covered with slimy material and was definitely separated from the surrounding bone.

About eighteen hours after the operation, the patient developed nausea, vomiting and vertigo, accompanied by a rotary nystagmus to the opposite side. During the severity of the attack, which lasted for about elght lays, the patient lay constantly on her left side with the eyes turned to the right. About the fifth or sixth day her hearing was tested and she was found to be stone deaf in her right ear. From that time on the patient made a rapid recovery and left the hospital at the end of sixteen days. Although still dizzy she was able to walk without assistance and each day showed a progressive improvement. It was felt, however, that we were dealing with an acute suppurative labyrinthitis which at any time might manifest itself by meningitis or brain abscess.

About six and one-half weeks after the radical mastoid operation, the patient began to complain of pain in the right side of the head, which became very severe at the end of three days. During the day she had a dull headache, but at night this became so intense that the patient was prompted to pull the hair out of her head in desperation. Definite tenderness of the skull on the right side above the back of the ear was elicited. A small drop of creamy pus was always present in the ear about the tympanic wall. There was no nystagmus and she past pointer spontaneously thrce inches to the right with the right arm and touched with the left. There was a definite tendency to fall backward with the eyes closed while standing upright.

A neurological examination was made by Dr. Marcus Neustaedter and he ieported as follows:

Blood pressure.-Maximum systolic, $118 \mathrm{~mm}$. Hg.; mininum dyastolic 90, pulse 108, temperature by mouth 99.5. Pupils, small; react promptly to light and accommodation. Right fundus, negative; left shows an old hemorrhage in the retina. Patellar reflexes, right, 1 plus; left, 2 plus. Pharyngeal reflex, absent. There is some swaying on standing with the eyes closed, but no true Rhomberg. A slight right facial paralysis, especially in the lower quadrant, is manifest, also a motor weakness in 
the right masseter and ptorigold muscles. The uvula assumes a diagonal position to the left. A left hemiparesis is apparent.

Diagnosis: Basilar meningitis and possibly a temporo-sphenoidal lobe abscess.

The cerebro-spinal fluid revealed a. slight turbidity, globulin 1 plus, Fehling 1 plus, Wassermann negative, cultivation negative, 500 cells to the cubic $\mathrm{mm}$. On the day following, the cells increased to 1040, and the fluid remained sterile.

On the same day she past pointed spontaneously with the left hand slightly to the right. 'The right ear remained stone deaf, good hearing was evinced in the left ear. No response was obtained from douching the right ear with cold water. ' On douching the lel't ear, no nystagmus or past pointing was elicited with the head upright, but with the head tilted backward, a large horizontal nystagmus to the right developed, but no past pointing was elicited in this position.

Dr. John MeCoy was called in consultation, and it was agreed between us that as long as the number of cells in the cerebro-spinal fluid had increased to such an extent in twenty-four hours, and the symptoms showed no signs of abatement, a labyrinthectomy was indicated immediately and was performed that day by Dr.. John McCoy with Dr. Mark J. Gottlieb assisting.

The Hinsburg operation was done. A very small dark area was found just above the prominence of the external semi-circular canal which looked like an area of necrosis. When a ring currette was passed over the internal tympanic wall from below upward, the face was seen to twitch several times, which indicated that the facial nerve was exposed either anomalously or as a result of bone destruction. The uncapping of the external semi-circular cunal was begun at the place where the fistula was found and in tusing the curette in a downward direction, a fairly large sized piece of bone was slivered of the internal end of the posterior bony wall of the external auditory canal and it was feared that either the facial nerve was exposed or completely severed. Fearing that possibly this patient might later show signs of' abscess of the cerebellum, a decompression of the posterior fossa was done at the same time. The day following the operation, a complete facial paralysis of the right side was found, with the exception that the patient sould partially close the right eys. On the same day, the number of cells in the cerebro-spinal fluid decreased to 450 , and from that time on, she made an uneventful recovery.

The facial paralysis has been treated ever since the patient has lelt the hospital, with massage and very light galvanic electric current and for the past three weeks a very definite activity of the muscles of that side has been apparent.

October 10, 1922. The muscles of the right side of the face are gradually regaining their power, and a complete restoration of their function is expected.

$$
\text { nTSCUSSION. }
$$

Dr. Kmbarsir (Broolklyn) inquired whether there was any relation between the blood pressure and the symptoms in Dr. Maybaum's case.

Dr. Hemze desired to know how the findings of the mastoid cavities compered to the X-ray intelpretations. As he believes that not only should the Radiologist have a complete history of the case before the picture is taken, but the operators should notify the Radiologist what what his diagnosis was (post-operative) also his operative findings. In this way only will the Radiographer perfect his opinion in reading future plates.

Dr. KING congratulated both Dr, Maybaum and Dr. Gottlieb on the excellent results secured in their cases. It was unusual to be able to show such patients in such excellent condition.

Dis. Mayiaun said that Dr. Gottlieb's case presented features of great interest. These cases of traumatic labyrinthitis require extreme care in the handling of them. Under ordinary circumstances it is a safe policy to leave well enough alone until definite reasons for interfering present 
themselves. He then cited a case of labyrinthitis following an acute middle ear suppuration. The patient, a boy of thirteen year's, was referred to him because of unilateral cleafness and discharging ear of three weeks' duration. A paracentesis had been done by the family physician, followed immediately by severe labyrinthine symptoms. 'The symptoms continued for five or six clays. On examination he was found to have total loss of hearing and vestibular function. There was also evidence of a mastoiditis present. When the father was told that the boy would have to be observed a few days and labyrinthectomy might be necessary, he took the child elsewhere. I was then informed that a simple mastoid operation was done, followed by development of a facial paralysis ten days later and a fatal meningitis shortly after. Labyrinthectomy ought surely to have been done with the onset of a facial paralysis in this case.

In these traumatic cases following an acute or chronic middle ear sup. puration it seems to be a better policy to wait for some clinical evidence of trouble before opening the labyrinth, and especially to be guided by the cerebro-spinal fluid examinations. Scme of these cases get well without operative interference. A labyrinthectomy should not be done during the acute stage of the disease.

Dn. War. Snosmisisters asked what were the indications for the labyrinthine operation, and cited a case recently observed. If a case showed all these symptoms,-- dead labyrinth, high temperature, meningeal symp. toms, cloudy fluid, etc., what was the rationale of waiting?

D1. Go'rLIEB, refer'ing to what Dr. Maybaum had said about traumatic cases and the necessity of waiting until meningeal symptoms arise, cited a case occurring in his private practice some yenrs ago in which a simple myringotomy was done. After 48 hours the patient developed acute suppurative labyrinthitis; and six months later he developed a meningitis and died in 24 hours. It had always been a question in his own mind whether it would not have been better had he opened up the labyrinth when the man had the initial labyrinthitis, rather than wait until the serious symptoms developed and there was no chance of saving life.

Dr. Gottlieb said that probably the explanation f'or the reduction of irritability in the other labyrinth was due possibly to the meningitis with a fibrinous exudate which organized and compressed some of the nerves, or involved the sheath of the opposite nerve, or possibly an involvement of some of the nerve tracts in the base.

Dr. MaYraunr. said that the reason for delaying operation in his case had been stated. Dach case was law unto itself as regards indications for operation. There are many cases where the same course followed as in this case would have resulted in a fatal outcome.

In this case the patient was seen when she had an acute suppurative labyrinthitis with beginning meningeal symptoms, high cell count and bacteria in spinal fluid. The spinnl flud was sterile at subsequent examinations. The case appeared hopeless at the begimning. To have operated then in all probability would have caused a spread of the infection from the labyrinth to the meninges. Repented spinal fluid examinations were made daily and showed a steady diminution of the number of cells. The polys later were replaced by lymphocytes, pointing to a recovery tendency. Each case must be considererl individually. In spite of rather stormy course during the first f'ew days, she showed a steady tendency to betterment. After an interval, she showed evidence of a recurrence of symptoms. It was then decirled to operate. The tendency, in handling these cases, is toward conservatism. As a rule a delay of ten days or two weeks is best so as to allow barriers to be formed against spread of infection.

\section{Acute Mastoiditis with lodoform Poisoning, Right Ear; Acute Mastoiditis} in Unusual Mastoid, Left Ear. Dr. Harold M. Hays.

There was nothing of unusual significance in the history of the case, but the patient was presented to give an opportunity to inspect the two mastoid wounds and to see the amount of destruction that can occur as the result of iodoform poisoning. 
Seven year's ago the patient had a typical mastoid infection, which was operated upon by an eminent otologist, who packed the wound with iodoform gauze. A few days later the patient's temperature rose to $105^{\circ}$, and nothing could be found to account for it. 'That state of' afiairs continued for ten days, the wound being packed with iodoform gauze, and no one suspected the cause of the trouble. After a weel the gauze was removed, and eventually l'ecovery took place, with a large wound extending far back in the heact. After six year's there is still in place which constantly scales and has to be cleared off. Last May the patient developed an acute infection in the other ear, and great care was exercised as to what was done. Dr. Hays waited for about ten days, before suggesting operation, testing the hearing as best could be done with the destruction and sup. puration that was going on; and at the end of that time it was explained to the patient that it would be better to operate on his ear rather than to run the risk of becoming totally deaf. 'The operation was performed early in June, and an unusually large mastoid cavity was exposed. An incision, extending backward two inches in length, was necessary. Learning from the patient that he was susceptible to most drugs, it was felt that neither Dakin's solution nor iodoform gauze could be used, so the wound was dressed with plain gauze packing; sufflcient to keep the edges open. At the end of the fourth or fifth day it was decided to try balsam of Peru, and that was the dressing used later on when the edges of the wound were drawn together by adhesive strips. The caustic stick was used on the granulations.

DISCUSSION.

Dr. DAvis said that as all knew he was an earnest advocate of the blood clot dressing following mastoidectomy, and this was a case in which it would have been very applicable. Particularly is this so in regard to the side first operated, for the iocloform packing, notwithstanding it had produced some iodoform toxcity, it certainly had rendered the wound cavity chemically sterile, therefore, by simply discontinuing the iodoform packing, thoroughly curetting the wound surfaces, admitting the blood-clot and closing the wound completely, in my opinion, healing would have been prompt, painless and without this great delormity. Again, no matter how large the mastoid cavity, the blood-clot fills the dead space which obviates the necessity of packing the wound.

Tn the second operation in this case, on the opposite side, the bloodclot dressing was just as strongly indicated and the marked deformities here shown. in all probability, would not have occurred; moreover, the patient would have enjoyed the advantages of quick healing and comfortable convalescence.

Dr. HAYs said that the case illustrated what sort of action one may get from iodoform. Dr. Davis and he agreed on what one of them called blood-clot dressing and the other called primary closure of the wound, and the two had often discussed the matter. He himself preferred whenever possible to close the mastoid wound, and he did so in the majority of cases. TTis per'sonal experience was that when one has so large a.cavity as this patient had it was almost impossible to bridge the gap, and if it was sutured the sutures were very likely to break down. for a great deal of tension had to be applied. As a rule, he did not believe in packing, but in this case it was necessary, but if it was necessary to use gauze, the paraffinized gauze would give a very much better result than the or: dinary kind. He was very loath to use iodoform gauze in any instance.

Otitic Meningitis (Serous) Following Acute Otitis Media and Mastoiditis; Mastoidectomy; Recovery. Dr. James Joseph King, New York.

S. L., a boy of six year's, was admitted to the New York Dye and War Infirmary, service of Dr. Robert Lewis, to whom $I$ am indebted for the pirvilege of this leport, on the 28th of February, 1.922, with the following histoly:

He was normal in health until five weeks ago, when he developed chicken pox, which was followed by mumps, and then measles. The left ear began to ache one week previous to admission to the hospital. The family physician was called and performed a paracentesis of the left 
membrana tympani. Following the incision small quantities of pus were discharged at various intervals. Hear'ing not impaired. Fever and headache for one week. No other symptoms.

Physical examination on admission: Temperature $104.4^{\circ}$, respirations 30, pulse 128. An anemic child, six years old, very ill in appearance, very restless and irritable, stiff neck, general hyperesthesia, has diminished knee jerks, positive Kernig on both sides, Oppenheim and Gordon suggestive, no Babinski present. 'The right ear and mastoid normal in appearance. The left mastoid swollen and tender throughout, canal full of thick, creamy pus, marked sagging of posterior superior wall. Drum reddened and bulging, pulsates through opening in drum membrane superiorly. Some secretion and slight obstruction in nose. Tonsils moderate in size, but intected. Tongue coated and dry. Heart, lungs, and kidneys negative.

Report of' Dr. Dixon on the radiographs of the mastoids is as follows: "I-Tas small clear right mastoid, sinus lorward, Left exceedingly cloudy, pneumatic, most all detail lost. Bone looks soft. Sinus in good position."

Bacteriological report showed a streptococcus infection, the type of which is not recorded.

Report- of spinal fluid on March 1: Clear, Fehling's positive, globulin positive, cell count 230 . Culture is negative for bacter'ia after 24 hour's' incubation. Blood culture also negative.

On this evidence a diagnosis was made of acute mastoiditis, left, with serous meningitis of otitic origin. Following the usual preparation a simple mastoid operation was performed by me on March 1, 1922. The cortex was fairly thin, revealing free pus and extensive granulations of hemorrhagic type underneath. The emissary vein was exposed on the cortex posteriorly. The antrum, not very deeply situated, was widely opened. The sinus was in good position with extensive perisinus involvement in a fairly large mastoid cavity: A posterior incision according to Whiting was made. A. large area of dura was exposed in the midale fossa showing slight injection of membrane and small petechiae. Dura was exposed by disease in the zygomatic region and there were granulations in this region. A very large area of dura was exposed in the region of zygoma and middle fossa, extending widlely beyond the area injected and covered with granulations. The horizontal incision was closed with metal clamps, but the posterior auricular wound was left wide • open. A Dakin's tube was sutured in and the wound dressed in the usual manner for the Dakin technique.

The temperature at time of operation was $105.6^{\circ}$. The child had the appearance of being very sick. The stiff neck, Kernig sign, and granulations and injection of the dura found at operation, lead us to believe that we were dealing with a serous or circumscribed meningitis, and that the child's life was saved by an early and complete operation.

The following notes were made: The blood count on March 1 showed red cells $4,000,000$, white cells 21,300 , small lymphocytes 1.7 , large lymphocytes 1.5, polynuclear's 81 . On March 1.0 blood examination shoved red $4,250,000$, white 27,000 , small lymphocytes 22 , large lymphocytes 4 , polynuclears 74. On March 21: Hemoglobin 80, red 4,000,000, white 5,200, small lymphocytes 40, large lymphocytes 2 . Polynuclear's 58.

The culture made at operation from the wound showed a mixed infection, including streptococci.

The daily examination of spinal fluid was as follows:

March 1. Spinal fluid clear, Felhling's positive, globulin positive, with 230 cells. The culture was negative for bacteria after 24 hours' growth. The blood culture was also negative at this time after 24 hours' growth. March 2. One day after operation. Spinal fluid clear, globulin negative, Fehling's positive, with a count of' 60 cells. Culture negative for bacteria after 24 hours.

March 3. Spinal fluid clear, Fehling's positive, globulin negative, cell count 20. Culture negative after 24 hours.

March 4. Spinal fluid clear, Fehling's positive, globulin negative, with a count of 14 cells, differential of which shows 50 per cent polynuclear 
and 50 per cent mononuclear. Culture negative for bacteria after $\mathbf{2 4}$ hours.

March 5. Spinal tluid cleal, Fellling's positive, globulin positive, cell count zero. Culture negative after 24 hours.

March 6. Spinal huid clear, Fehling's positive, globulin positive, cells 12. Culture negative after 24 hours.

March 22 . Blood culture negative after 24 hours.

jeye grounds negative at all examinations.

'Temperatule al'ter operation receded from $105.4^{\circ}$ to $103.4^{\circ}$ with a slight elevation in the night to $104^{\circ}$. From that it gradually came down to 101 on the third day. On the night of the fifth day the temperature rose again, however, to $105^{\circ}$.

On March 10 he complained of pain in his' right foot and leg and on March 11. I made the f'ollowing notes: "Pain in right leg at knee. It is contracted, and boy complains of pain when it is extended." Dr. Lew is examined him with me and he was suspicious of a beginning psoas abscess. On the 11th we asked Dr. Eugene Poole to see him and he made the following notes: "Ablomen seems negative, flexion of hip and tenderness around hip suggests metastatic abscess, likely in the joint." He suggested $X$-ray and watching for definite evidence which would demand an incision. We watched him as directed by Dr. Poole and no abscess developed and I think we were justifled in believing that this manifestation was just an evidence of sepsis manifested in this part from the original focus in the liead. This gradually disappeared as general improvement advanced.

The posterior wound which had been left wide open was very slow in healing. On the 14th of March the ear canal was dry and the Dakin tube was removed. The wound was filled with excessive granulations. On the 23 r'd we decided to take him to the operating room, curette the granulations, and make an attempt to close the posterior wound, and to remove the tonsils and adenoids. I curetted the wound and made an attempt to close the wound with silk-worm gut sutures. There was such a profuse hemorlhage at this time that I decided not to remove the tonsils and adenoirls. The sutures did not hold, but the wound seemed to improve after this. On the 30 th of March he wis again taken to the operating room and the tonsils and adenoids were removed, and another attempt was made to close the posterior wound. This was not entirely satisfactory, but from this time on he continued to improve until the wound was well closed and epidermitized entirely.

It was deemed advisable to remove the tonsils and adenoids at this time because of the generally septic appenrance of the child and its manifestation as the pains in the leg, etc.

He was discharged from the hospital on April 6. The wound soon became completely healed following a fow dressings in the dispensary.

It was my pleasure to report before this Section a similar case on FebI'uary 10, 1922. This was an adult with less marked ear and meningeal symptoms. His spinal fuid, however, showed 1,000 cells to the count. He also made a complete recovery following a simple mastoid operation and large exposure of dura in the middle f'ossa, ns has been recommented by Dencl.

\section{conclustons.}

1. Serous or circumscribed otitic meningitis is an early stage of septic meningitis.

2. The early removal of the focus of infection in the ear by mastoidectomy with large exposure of dura offers the only hope for saving the patient's life.

Dn. HArs said that these cases of possible meningitis in connection with acute mastoditis were very interesting, and sometimes very baffling, and cited a case of acute mastoid with every symptom of meningitis. The patient was taken to the hospital and the mastoid was exposed, and later made an uneventful recovery. Apparently all the symptoms were due to an erosion of bone, and disappeared immediately after free ex- 
posure of the dura and discharge of pus. Whether such cases are really cases of serous mennngitis it is difficult to say. A year ago he reported a case of double mastoid. 'The wound did not henl on one side and a second operation had to be performed. On the second day the temperature jumped to $106^{\circ}$, with every symptom or meningitis. A lumbar puncture was done, revealing fluid uncler pressure, a cell count of 2200 lymphocytes was tound, and a hopeless prognosis was made; the culture of the spinal fluid hat not been received $u_{1}$ to tnat time. Atter the consultant had left the mother asked if she could get 11 a Christian Scientist, and was told it made no ditterence what she did. The following day the child was sitting up in ber, and later lett the hospital per'lectly well.

Another class of cases have a d'elation to this:-cases of children who have had acute middle ear symptoms, but whose ear drums were never opened. Suddenly some weelss later they developed symptoms of meningitis. In two such instances the mastoids were opened and found in fairly good condition with a little serous fluid in the antrum, and both children died. In other cases where there has been little or no discharge from the enr and where the mastoid symptoms have been very much obscured, it has been difficult to clecide whether or not to operate, for one would hesitate to say that an operation had better be done for fear a meningitis might develop; yet such cases as this of Dr. Fing's made one think very seriously.

Dr. Herzic congratulated Dr. Ising on the successful outcome of his case. The point that impressed him was the repeated spinal punctures, which doubtless had a great deal to do with the clearing up of the spinal count and meningitic symptoms. In his opinion resort should be more frequently made to that procedure in meningitis.

Dr. KopersKy said that the case which Dr. King presented with a distinct pathological finding in the middle ear and distinct foci was one thing; with the case reversed, such a case as Dr. Hays spoke of, with very little findings in the middle ear and very little serum, and the condition so slight that the middle ear was not even opened, it was a very different thing. Here vere two pictures; in the one, a prodromal period and then a meningitis; in the other, a distinct lesion in the ear, the condition in the middle ear advancing and by contiguity involving the dura, and seen by the surgeon when the granulations were present on the dura. We all regard granulations such as these as protective in nature. One has every reason to expect recovery in such a type of case; and very little reason to expect recovery in Dr. Hays' case, for there nature made no effort to protect. That seemed to be the explanation. This comparison was not made in any spirit of detraction in regard to Dr. King's case, but was made to call attention to the varying types of cases discussed.

Dr. KING said that there was a very definite pathology in the middle ear, a perisinous involvement, and expostire of the dura by disease, with granulations on it; and the inclications were clear for what was done. Dr. Lewis was consulted and agreed thoroughly in what was done. The spinal puncture helped greatly in the case; there were six spinal punctures in this instance; the other case had not so many, but he got well just the same.

\section{Successful Blood Clot Dressing Secondary to Unsuccessful Carel-Dakin} Dressing in Double Mastoidectomy. Dr. George $\mathrm{E}$. Davis.

'E. F. G., age 11 months.' Referred to me April 19, 1922.

History:-Seven weeks previously developed cold and was treated by a pediatrist. During this period patient ran temperature varying from $101^{\circ}$ to $106^{\circ} \mathrm{Ir}$. and the case was diagnosed as pnemonia. One week before I saw the patient she came under the care of a prominent otologist, who did a double mastoidectomy and instituted the Carrel-Dakin aiter treatment. Under this treatment the patient did not progress favorably, the temperature fluctuating between $102^{\circ}$ to $104^{\circ}$, and at the end of a week consuitants diagnosed pneumonia as a complication, alleging this as the cause of the continued fever:. On this account the parents were advised that it would be necessary to transfer the patient to another hospital-Bellevue. However, the parents took the patient home and called 
their family physician, who, after a careful examination, stated that the lungs were not involved, but believed the condition of the ears were responsible for the hyperpyrexia, etc.

I saw the patient the following day, which was the sixth day subsequent to the original mastoidectomies. After removing the dressing and the tubes through which the Carrel-Dakin solution was applied, I thoroughly curetted both mastoid wounds-admitted the blood-clot on both sides and closed the wounds entirely. A single, sterile, thin ganze strip was inserted in the auditory canal on either side and a light sterile gauze dressing adjusted over all. The outer gauze dressings and gauze canal drains were changed daily. Succeeding the blood-clot dressing the fever dropped to about normal the following day and remained so without other treatment. In three days primary healing of the mastoid wound was complete on the left and in five clays the same hapny results on the right, with both canals dry and which have remained so.

Of course no general deductions can be made on a single case, but it is reported for what it is worth and as a confirmation of similar lesults obtained in a very great majority of cases where the blood-clot is employed primarily. Moleover, in the case reported the blood-clot dressing proved successful in the patient's home with a single nur'se, whereas the Carrel-Dakin was a failure in a well regulated hospital. DISCUSSION.

Dh. HAYs said that he and Dr. Davis were very good friends and their discussions and controversies were merely over technical names, etc. He was much pleased that Dr. Davis had brought up this subject, for he had spoken of the non-success of the Carrel-Dakin solution. Since the war all of the men had used the Carrel-Dakin solution and Dichloramine-T, and many had come to the conclusion that the results from its use were often exaggerated. For many year's he has closed mastoid wounds, excepting at the lower angle where he places a small strip of gauze, and examines the wound every day and expresses material from it; this material is usually sterile. Once or twice he had found it necessary to open the wound. One of these was a case of erysipelas, but he had never found a blood clot in the mastoid wound. One may have a clot at the close of an operation, but you get a fibrinous mass which does not fill the cavity completely, and it was diffeult to see how it could be called a blood clot. His own idlea was that a bridge of skin forms over the cavity which flls with granular tissule. In the cases which break clown,-in children especially with lowered vitality,-there is probably a nasty slough, with granulations, etc., which are very difflult to overcome. Sometimes one does succeed in sterilizing the cavity with Carrel-Dakin solution through a tube, or with Dichloramin-T, but if you keep on using the antiseptics for a length of time, a severe renction is created which interferes with the welfare of the patient Recently an eminent colleague stated that he had had a number of cases treated with Carrel-Dakin with marvelous results, but comparing these cases which have been partially closed with his cases they bear the comparison very well indeed. Dr. Hays feels that the healing process has been suffiently rapid, wounds healing in from four to fifteen days, by emptying a primary suture and nothing more.

Dr. Go'stries said that at the New Yorks Infirmary they had had a good deal of experience with the Carrel-Dakin solution, but the method would come to glief if the solution was not properly prepared. In this case presented by Dr. Davis, it was the preliminary treatment that prorluced the complete healing after he closed the wound : for in the flrst instance the wound had at least six days to go before Dr. Davis had the oppottunity of operating. and during those six days a protective barrier was set un: after the injury of the operation had subsirler the number of bacteria decreased. as in all wounds that have been flrained. anr it did not slueak so much in favor of the blood clot method that Dr. Davis arlvocated as one would presuppose. Very often one would get with the CarrelDakin a breaking down of the edges of the wounr, probably due to improperly prepared solution. He was sure that those who dressed the cases at the inflrmary knew more about it than did the actual attendants, 
and he hoped that either Dr. Shannon or Dr. Hogan would tell about their experience in using the solution in clressing mastoid wounds.

Dr. C. W. Shavion sald that he had used the solution for two or three months under the best technique established at the infirmary. The fir'st few cases had very poor results, but atter the teclinıque and solutions were thoroughly established the cases became very much improved and, as a rule, the results were very satislactory; but, as Dr. Gottlieb said, the technique and operator make for success and all of these things together make it sometimes difficult to tell whether it is the solution that gives the result or not. The best result showed solid healing with dry canal -and posterior wound in seven to ten days, while nearly all cases were discharged dry within two weeks. But, as in general surgery, the Carrel technique and Dakin's solution, both being so exacting, that its practicability became the main objection.

Dr. IIogan said he thought the children healed as well one way as the other, especially at the age of five or six year's; an adult would usually be discharged in 12 days, and the other's would be held much longer.

Dr. Koperzkr said that some years ago, when Sprague first brought out the blood clot treatment, and this was followed by Reik of Johns Hopkins, a wave of enthusiasm for this treatment of the post-operative wound spread over the country. Quite a series of cases were thus handled at the Manhattan Eye, Ear and Throat Hospital. Some of these crtses did well and were discharged. Of the cases which he had operated and handled in this way, Dr. Kopetzky said that every single one of them eventually returned and requil'ed a secondary oper'ation.

Dr. Kopetzky said that this was a clinical meeting where every one gave his own personal expelience; but if one really wanted to compare different techniques, it should be done by the same man's using the dil.ferent kinds of techniques upon similar types of cases. Then, only, would a proper judgment be rendered. Dr. Reik had opportunity to see, upon one occasion, some of Dr. Kopetzky's cases and then told him that such were not considered by him (Reik) as suitable for the employment of his method. In other words, it developed that the speaker and Dr. Reik were not using the method upon similar classes of cases. So if Dr. King or Dr. Davis or Dr. Hays got good results, and you do not, you must be quite sure that the cases submitted to the test of treatment were similar.

Anything that would shorten the after treatment in mastoid cases would prove of great value and was of interest. The Section was grateful to Dr. Davis for presenting this subject.

Dis. MAYmun said that whatever criticism is to be directed at the method of healing mastoid wounds advocated by Dr. Davis, 'he is certainly to lje congratulated upon the results obtained in the case he presented. Dr. Maybaum said that he had never been able to get away from the notion that the blood clot method offered, in most cases, an ideal medium for growth of bacteria in a mastoid wound. No matter how careful and thorough one may be in performing a mastoid operation, one surely can never completely sterilize the wound. The rationale of the method had never appealed to him.

He had used the Carrel-Dakin method in: a number of instances, but it did not appeal to him. He knew of cases where its use had been followed by chronic discharging middle ear's with large perforations. An advantage of the method is the comparative painless character of the dressings. The labyrinthectomy case presented this evening was dressed by CarrelDakin method. It was very satisfactory in this case. By the old method, the dressings woulkl have been more painful and prolonged.

The last word has not been said on the subject of healing mastoid wounds. Thère is no question that Dr. Konetzky is correct in the view he expresses of the many factor's entering into the healing of a mastoid wound. A thorough operation is essential, no matter what method is used. The old method of gauze packing ought to be discarded; it is an admirable means of prolonging healing and continuing the infection. Almost closing the wound and using a rubber tissue strip is a far better method than packing with gauze. 
D1. Doughlisty said he wished to say a word on the wisdom of discarding packing. The mastoid wound is nothing more or less than 'any other' abscess cavity; and the general surgeons laugh at the way the otologists treat mastoid wounds as open wounds. The surgeon will remove a chain of glands from the neck, put in a rubber tissue drain, and sew up the incision; in a week, the case is dischar'ged, healed.

He also wished to emphasize what the Chairman had said. He also was one of those who had religiously tried the blood-clot methos of heal. ing; he had always envied Dr. Davis in his results, but had never been able to obtain them himself. To his mind, there was always present the danger of making a perfect nidus of infection; no one is absolutely perfect, at all times, in the performance of a mastoid operation; the wound may be still slightly septic and the clot form a fine culture medium for the bacteria. Dr. Dougherty said he had tried the Carrel-Dakin method and would advocate it in certain cases. In those cases of sinus-thrombosis and jugular resection where there is an extensive infection in the surrounding tissues, there is nothing better, although it does sometimes macerate the edges and cause considerable irritation. Of late, he had been sewing up the mastoid wounds and placing a thin drain in the lower angle of the wound; if he had found it necessary to use anything in the line of the Carrel-Dakin dressing he had used Dichloramine-T in chlocozone, saturating the drain with the solution.

Dr. Davis was decidediy to be congratulated on the results he had gotten and it was no reflection on his work to say that in many cases it was not so much the personal equation of the operator as the personal equation of the case. That every case operated upon was not, in the strict sense of the word, an operable case, is the experience of every otologist; the question of operation has been a doubtful one and the patient has been given the benefit of the loubt. In these cases the wound is not septic and heals readily under any kind of treatment.

Dr. Divis, closing the discussion, said that the case was reported for what it was worth and to contrast the blood-clot method with the CarrelDakin treatment following mastoidectomy, particularly in children, and he was pleased with the interest and liberal discussion elicited.

The after treatment of mastoidectomy is about twenty-five years behind the times. That statement was not meant as a reflection on any one, but as he had stated in previous discussions the subject was a very broad one and he is convinced that the profession will eventually realize its mistake in continuing antiquated methods, and that the aclvantages of the blood-clot method will finally force its acceptance.

One of the gentlemen raised the question whether the Carrel-Dakin treatment six days preceding the blood-clot dressing facilitated the healing. Perhaps so, but the time of healing in this case is not unicue, though the blood-clot was secondary to the Carlel-Dakin treatment. Other cases have healed quite as readily, notably in the young, where the bloodclot was used primarily. Moreover, during the period of' the Carrel-Dakin treatment in this case, the hyperpyrexia continued and the general condition was not good at all. These symptoms were regarded not due to the ear conditions, but to a complication diagnosed as pneumonia, and - on this account the parents were requested to transfer the patient to another hospital or his home. With the substitution of the blood-clot method for the Carrel-Dakin treatment 1 am happy to leport that all unfavorable symptoms promptly subsided and in a very few days both sides were completely, healed, canals dry, and have remained so, notwithstanding the child has a mass of adenoids in the pharyngeal vault and hypertrophied tonsils.

Dr. Kopetzliy, in his discussion of this case, has seen fit to make an invidious comparison of the diagmosis and character of the cases operated by himself' and a distinguished confrere, which' I must say I feel is altogether unfair and unjust. Not that Dr. Reik needs any one to champion his part, for his ability and authority as an author, teacher and surgeon is well known and his work compares favorably with the best. I am quite sure his statistics are based on authentic cases. 
Dr. Kopetzky requested me in closing the discussion to report how long I had lollowea ny cases treated by tile bloon-ciot methou and the percentage of secondary operations. For his inrormation and to convince hum that this technique is adapted to geuine mastolditis (even with complications), 1 am preased to cite a case reported six years ago, in a man about torty-hve year's or age', an alcoholic, syphilitis and general condition poor, one wnom, at the Polychinic Hospital, I did a leit mastoidectomy complicated with a very extensive sub-periostal abscess, a perisinal abscess and smus thrombosis, and after packing the wound five days with iodolorm gauze, a bloocl-clot was admitted and the wound closed. Five days later the wound was healed save in the lower half incl, where there was a partial breaking down of the clot, but in flve or six days more healing was complete and with very slight deformity. This man was well at the end of two years, when I lost touch with him, as he was a traveling actor. Certainly this case demonstrates that the bloodclot is suited to severe cases, not merely for mild or unauthentic ones. Noreover, the blood-clot technique, in my experience at lenst, does not require secondary operation more frequently than the older methods.

Dr. Dougherty's remarks in regard to the splendid manner in which general surgeons handle bone-abscess cases were apropos and $I$ agree with him that our work, in comparison, for the most part in such cases, should make us ashamed of ourselves. Halstead, that master surgeon recently passed beyond, Oeschner and other's have illumined the way if we but had the courage to follow!

Dn. Kopersir expressed the hope that no one had interpreted his remarks as being in any way a criticism of Dr. Reik, for he had never questioned his ability as a conscientious diagnostician and surgeon, and liad the deepest respect for him. It was simply because of his deep friendship for him that he had taken the liberty of citing his name as an example.

\section{Double Mastoiditis Pneumonia and Sinus Thrombosis in Baby Fifteen Months Old. Dr. J. Morrisset Smith.}

The complications occurring in the following case mate it very difficult to decide just what course would offer the patient the best chance for' recovery.

The baby was admitted to the hospital with a double mastoiditis, a simple masto:d operation was done on the left side, mastpid cells were broken down filled with f'ree pus, no exposure of sinus or dur'a, usual dressing, incision was done on the right drum and the patient returned to bed.

The temperature gradually came down and progress seemed satisfactory. The right ear was discharging freely, but operation was not deemed necessary.

One week later temperature suddenly jumped from $1001 / 2^{\circ}$ to $1058 / 10^{\circ}$. A total white cell count showed 16,000 cells CnM.M., and blood culture showed in twenty-four hours filteen colomis of hemolytic streptococci; the consulting internist reported a pneumonia right side. The problem here was very complex in lieu of the positive blood culture, double mastoid infection and the pueumonia.

The baby was taken to the operating room and right mastoid was thoroughly cleaned out, the lateral sinus was exposed and apparently normai. The left mastoid was then reopened, the lateral sinus exposed; there was no evidence of a complete clot, but there was a small dark looking spot on the sinus wall just below the knee. The sinus was completely uncovered from above the knee down to the bulb, then, due to pneumonia and time consumed by operation, the patient was returned to ber, decirling that it would be better to defer the sinus operation for a day or two. The next day the patient's temperature was $1044 / 10^{\circ}$; a second blood dulture was taken which was negative at the end of 24,48 and 72 hour's.' Improvement continued, temperature came down and an uninterrupted recovery followed. The most plausible explanation would seem to be that the infection in the sinus was intramural and from this point the organ- 
isms were released in the blood stream, giving a positive culture, following the removal of the bone over this area, the infection remained localized in the sinus wall and was able to take care of itself.

DISCUSSION.

Dr. Gorrulise congratulated Dr. Smith on the results obtained in so serious a complication of conditions. Refel'ring to the matter of determining which, if not both, sinuses are affected, he cited Dr. Kopetzky as telling him of a case of double mastoiditis in which he was thinking of the possibility of' determining the number of bacteria in the blood com. ing from the internal jugular, but said he had never heard anything more of that particular. method of determining the location of the infection. It would be very interesting to hear from Dr. Kopetzky on that point.

Dr. MayiBaun said that Dr. Smith was to be congratulated upon his good judgment and conservatism in this case. Young children do not stand jugular and sinus any too well; a complicating pneumonia makes the condition extremely dangerous and a poor operative risk.

Undoubtedly there are cases of sinus phlebitis without clot formation giving rise to sepsis, as in this case. A sinus phlebitis with clot formation sometimes takes care of itself because of the bactericidal properties of the blood.

Where both mastoids are involved and the question of which jugular to tie comes up-unless definite difference in the appearance of the sinuses is present-it is far better to do nothing. He cited a case seen at Manhattan Hospital some year's agc-a case of Dr. Friesner-an adult with double mastoiditis-positive blood culture-septic temperature. Nothing definite could be determined by exposing both sinus plates. The man developing multiple abscesses in the muscles and subcutaneous tissue. After a very stormy cour'se he recovered without jugular or sinus operation.

Dr. Korletzis , replying to Dr. Gottlieb's question, said that a case of double mastoid and sinus thrombosis with a positive blood culture presented a very serious and pressing problem, but one which had to be faced at the bedside; and at the time to which the Doctor referred he had been worlking on that particular problem. In order to make the test, one must have a very expert hematologist and must with absolute precision take from both jugulars equal quantities of. blood; then count the colonies growing on the blood taken from both sides and base the results on the conclusion obtained. On the side where the thrombosis is you should get more colonies than from the opposite side. The logic of the matter was open to many fallacies, however, - the fact that bacteria grow in the blood stream of a case is going bad is one, and secondary foci is another,- but the test offer's one method of approaching a solution. It is not conclusive. There were other ways-listening to the blood stream bruit, etc. The best determination was to be found in the study of the original pathological lesion at operation; the side that presented the greatest amount of lesion, the greatest destruction, is the one where the sinus would be suppused to be involved.

Dr. Kopetzky said he did not wish to be understood as advocating this differential counting as a means of diagnosis.

Dr. Smrrtr said that in this particular case at the primary operation there was no exposure of the sinus; but "of course there was a double mastoid infection and this, together with a positive blood culture, made it difficult to decide what should bé done. Had it not been that a pneumonia also was present, he would have ligated the sinus and cleaned out the jugular, but there was no doubt that if you were to ligate both jugnlar's the patient would die. In this case, after the sinus was exposed, a dark spot was found' on the wall, but no clot. Where you are confronted with a double mastoiditis and a positive blood culture, the best proceedure is to uncover the lateral sinuses, first one side and then the other, and you are very apt to determine by inspection and palpation the location of the lesion. When it comes to taking blood from the jugular, it must be remembered that you may have a completely clotted sinus and get no. organisms in the culture, so that should the clinical symptoms of a sinus thrombosis continue this factor must not be overlooked. 